Published in final edited form as:

J Nanopart Res. 2014 June 1; 16(6): . doi:10.1007/s11051-014-2379-1.

\title{
Toxicity of polymeric nanoparticles in vivo and in vitro
}

\author{
Nadine Voigt, \\ Institute of Medical Psychology, Otto-von-Guericke University, Leipziger Str. 44, 39120 \\ Magdeburg, Germany \\ Petra Henrich-Noack, \\ Institute of Medical Psychology, Otto-von-Guericke University, Leipziger Str. 44, 39120 \\ Magdeburg, Germany
}

Sarah Kockentiedt, Institute of Process Engineering, Otto-von-Guericke University, Universitätsplatz 2, 39106 Magdeburg, Germany

Werner Hintz, Institute of Process Engineering, Otto-von-Guericke University, Universitätsplatz 2, 39106 Magdeburg, Germany

Jürgen Tomas, and Institute of Process Engineering, Otto-von-Guericke University, Universitätsplatz 2, 39106 Magdeburg, Germany

\section{Bernhard A. Sabel} Institute of Medical Psychology, Otto-von-Guericke University, Leipziger Str. 44, 39120 Magdeburg, Germany

Bernhard A. Sabel: imp@med.ovgu.de

\section{Abstract}

Polybutylcyanoacrylate nanoparticles (PBCA NPs) are candidates for a drug delivery system, which can cross the blood-brain barrier (BBB). Because little is known about their toxicity, we exposed cells to PBCA NPs in vitro and in vivo and monitored their life and death assays. PBCA NPs were fabricated with different surfactants according to the mini-emulsion technique. Viabilities of HeLa and HEK293 cells after NP incubation were quantified by analysing cellular metabolic activity (MTT-test). We then repetitively injected i.v. rhodamine-labelled PBCA NP variations into rats and monitored the survival and morphology of retrogradely labelled neurons by in vivo confocal neuroimaging (ICON) for five weeks. To test for carrier-efficacy and safety, PBCA NPs loaded with Kyotorphin were injected in rats, and a hot plate test was used to quantify analgesic effects. In vitro, we found dose-dependent cell death which was, however, only detectable at very high doses and mainly seen in the cultures incubated with NPs fabricated with the tensids SDS and Tween. However, the in vivo experiments did not show any NP-induced neuronal death, even with particles which were toxic at high dose in vitro, i.e. NPs with Tween and SDS. The increased pain threshold at the hot plate test demonstrated that PBCA NPs are able 
to cross the BBB and thus comprise a useful tool for drug delivery into the central nervous system (CNS). Our findings showing that different nanoparticle formulations are non-toxic have important implications for the value of NP engineering approaches in medicine.

\section{Keywords}

Blood-brain barrier; Retina; In vivo imaging; Nanotoxicology; Polybutylcyanoacrylate; Nanoparticles; Analgesia; Environmental and health effects

\section{Introduction}

Nanoparticles (NPs) hold the promise to be a universal tool for drug delivery into the brain. This has been a research topic for more than a decade, but unfortunately, successful pharmaceutical developments are still rare. This can be explained in part by toxicity concerns which have not been considered sufficiently.

In fact, during the recent years, we have witnessed an increasing public concern regarding the potential toxicity of NPs (De Jong and Borm 2008), and there are ongoing international efforts to develop tests to evaluate the safety and tolerability of NPs after exposure of biological systems, and the mechanisms of action are now partially understood (SCENIHR 2006).

The focus of these efforts has been on the possible toxicity of NPs following inhalation or food intake, and therefore predominantly lung and gastroenteral toxicities have been investigated. However, only a minority of the studies focused on brain cells. It was suggested that-in contrast to chemical toxicity, where purity and concentration are the main determinants - in the area of 'nanotoxicity' also particle size, shape, surface and charge need to be taken into account. The literature shows that NPs can cause inflammation, DNA damage and destruction of membranes and production of reactive oxygen species. However, specific hazards are associated with specific particles, i.e. DNA damage and production of reactive oxygen species are predominantly associated with metal (oxide) NPs, whereas, membrane leakage has been observed after application of polymeric NPs. To test nanotoxicity, in vitro test systems have been largely used, and the MTT-test of viability is recommended for this purpose. However, in vitro experiments may be insufficient as a strategy for testing the safety of NPs, and data regarding the correlation of in vitro and in vivo studies are entirely missing (Dhawan and Sharma 2010; Ai et al. 2011; Zhao and Castranova 2011; Elsaesser and Howard 2012; Sharma et al. 2012; Love et al. 2012; Nunes et al. 2012; Nyström and Fadeel 2012).

As it was also shown that inhalation of NPs through the nose leads to an entry of the particles into the brain via transport along the olfactory bulb (Oberdörster et al. 2004), an important issue is to probe the potential neurotoxic effects of NPs on the central nervous. This issue is of interest for the following reasons: (i) the unintended passage to the brain by inhalation, and (ii) the intended use of NPs to transport active agents across biological barriers (Gabathuler 2010), especially to the retina and brain, for diagnostic and therapeutic purposes.

J Nanopart Res. Author manuscript; available in PMC 2015 September 27. 
Yet, despite a growing effort to study NPs, the crucial issue of potential central nervous system (CNS) toxicity has not been addressed sufficiently so far. In addition to potential CNS side effects of NPs released into the environment and their unintentional inhalation, potential neurotoxicity of NPs in the nervous system is a key issue for the development of carriers which transport drugs across the blood-brain barrier (BBB). The inability of most pharmaceuticals to pass the BBB is a major challenge for drug development to treat diseases of the CNS, and NPs may offer a new solution. The WHO rates CNS diseases as the most important medical concern of this century, and so finding new drug delivery systems which are not only effective but also safe is a critical step in this effort. As we will later show, repeated exposure of rats to Polybutylcyanoacrylate nanoparticles (PBCA NPs) in vivo did not reveal toxic effects, and thus, with the doses used, they appear to be safe.

\section{Materials and methods}

\section{Animals}

Male hooded rats (CrL: LIS) were purchased from Charles River at 9-10 weeks of age and kept at a 12-h light/dark cycle, ambient temperature $24-26{ }^{\circ} \mathrm{C}$ and humidity of $65 \%$. Food and water were available ad libitum. Narcosis was induced by i.p. injections of Ketamine $(75 \mathrm{mg} / \mathrm{kg}$ ) and Xylazine $(10 \mathrm{mg} / \mathrm{kg})$. The rats were randomly assigned to one of four groups: (i) ONC group (optic nerve crush after baseline imaging; for control of neuronal death detection), (ii) sham control-group (i.v application of Rhodamine 123 (0.36\% in saline) every week) and two NP-groups, (iii) Tween80-group (i.v application of Tween80Rho123-PBCA NPs every week), and (iv) Tween80-SDS-group (i.v application of Tween80-SDS-Rho123-PBCA NPs every week). All NP variations were injected intravenously via the tail vein with a single dose of $0.1 \mathrm{ml} / 100 \mathrm{~g}$ body weight using a preimplanted catheter (BD Adsyte Pro, 22 G, Heidelberg, Germany). During the whole experiment, the behaviour, weight and appearance of the rats were monitored. At the end of the experiment, the animals underwent transcardial perfusion, and their hearts, livers, kidneys and brains were all removed, frozen at $-80^{\circ} \mathrm{C}$ and examined after histological processing. The experimental design is shown in Fig. 1.

For the hot plate test, Kyotorphin was used as an analgesic test substance which was injected into the tail vein of rats without narcosis using the following formulations: (i) Tween-PBCA NPs Kyotorphin (incorporated) $10 \mathrm{mg} / \mathrm{kg}$, (ii) Tween-PBCA NPs Kyotorphin (adsorbed) 10 $\mathrm{mg} / \mathrm{kg}$ or (iii) Kyotorphin alone $10 \mathrm{mg} / \mathrm{kg}$.

\section{In vivo confocal neuroimaging (ICON)}

Labelling of retinal neurons [retinal ganglion cells (RGC)] was carried out by injecting $2 \mu \mathrm{l}$ MiniEmerald (dextran, fluorescein and biotin; Invitrogen) into both superior colliculi. To this end, the cranium was exposed and holes drilled at the following coordinates (oriented to bregma/lambda): post. $-6.9 \mathrm{~mm}$, lateral $1.2 \mathrm{~mm}$. The tracer $(0.5 \mu \mathrm{l})$ was injected at each of the following depths below dura: 4.0, 3.5, 3.0 and $2.5 \mathrm{~mm}$.

To follow the fate of RGC after NP application, ICON was carried out every week before the next dose of NP was applied. To this end, the eyes of anaesthetized rats were treated 
with Neosynephrine-POS $5 \%$ (Ursapharm Arzneimittel GmbH, Saarbrücken, Germany) and Vidisic optical gel (Bausch\&Lomb, Berlin, Germany). The rats were then fixed under a confocal scanning microscope (LSM 5 Pascal, Zeiss GmbH) with the eye positioned underneath the objective lens. A -80 dioptre plano-concave lens (KPC-013, Newport $\mathrm{GmbH}$ ) was placed onto the cornea.

\section{Image analysis}

For morphological analysis in vivo, we defined retinal areas which, in repeated imaging, could be relocated using blood vessel morphology and followed them over the whole experiment. The number of fluorescent retinal neurons at baseline was quantified, and we observed these cells at the later data collection points. The cell soma diameters and the cell fluorescence intensity were measured with the AxioVisionRel. 4.8 programme (Zeiss, Jena) in each cell and averaged automatically for calculation. The limit between the fluorescent cells and the background was detected automatically using a grey scaling method ( 8 bit accuracy).

\section{Optic nerve crush (ONC)}

To induce neuronal injury, an ONC was carried out on both eyes as previously described (Sautter et al. 1991; Sautter and Sabel 1993). To this end, a lateral canthotomy was made from the orbita following an incision of the conjunctiva lateral to the cornea. The retractor bulbi muscles were separated, and the optic nerve was exposed by blunt dissection. To crush the nerve, a calibrated forceps (Martin Instruments, Tutlingen) was used for $30 \mathrm{~s}$ at a distance of 2-3 $\mathrm{mm}$ from the eye. The jaws of forceps were set at $0.2 \mathrm{~mm}$ apart in 'restposition' which produced a moderate injury. After the surgery, an antibiotic eye ointment (Aureomycin) was applied.

\section{Cell culture}

To analyse the toxicity of PBCA NPs, a cell viability test (MTT-assay) was carried out. Two different cell lines [HEK293T/17 (human kidney cells) and HeLa (human cervix epithelial adenocarcinoma cells)] were used to control for a cell line-specific reaction. 40,000 cells were cultured in a 96-well plate in DMEM for $24 \mathrm{~h}$ before being incubated with different NP concentrations (non-fluorescent) or with medium (DMEM) only (control). After another 24 $\mathrm{h}$, the cells were washed, the negative control was incubated for $10 \mathrm{~min}$ with $96 \%$ ethanol, and then incubated for $4 \mathrm{~h}$ with the MTT salt $(0.5 \mathrm{ml} / \mathrm{l})$. At the end of the experiment, the change of colour was analysed with a fluorometer (OPSYS MR, $560 \mathrm{~nm}$ ).

\section{Nanoparticle production}

PBCA NPs were synthesized by a mini-emulsion polymerisation process. In brief, an O/W (oil-in-water) mini-emulsion was made from two solutions: an aqueous phase (solution I) and an oil phase (solution II). For solution I, surfactant and optional Rhodamine123 or Kyotorphin were dissolved in phosphoric acid, and soybean oil (Roth Karlsruhe) was mixed with $n$-Butyl-2-cyanoacrylate (BCA, Roth, Karlsruhe) monomer for solution II. Solution (I) was added to solution (II) just prior to ultrasonication. The two-phase mixture was sonicated with a Bandelin Sonopuls HD 70 sonifier (70\% amplitude) for 4 min under ice cooling. At 
$30 \mathrm{~s}$. thereafter, the polymerisation was induced by pouring the mini-emulsion into ammonia solution with stirring using a magnetic stirrer. After $5 \mathrm{~min}$, the $\mathrm{pH}$ was adjusted to 7.0. The resulting PBCA NP suspension was kept at $6{ }^{\circ} \mathrm{C}$ under protection from light until further use in the experiment. For details see Tables 1 and 2.

The materials and the respective suppliers were $n$-Butyl-2-cyanoacrylate monomer (Henkel AG \& Co.KG, Düsseldorf, Germany), Lutrol F68 (Poloxamer 188) (BASF Ludwigshafen, Germany), sodium dodecylsulfate (SDS; >99\%) (Roth, Karlsruhe, Germany), Tween80 (Polysorbate80) (Roth, Karlsruhe, Germany), soybean oil (Ph. Eur.) (Roth, Karlsruhe, Germany), Rhodamine 123 (>98 \%) (Biotium), Dextran 70000 and DEAEDextran (each from Sigma, Taufkirchen, Germany), and Kyotorphin (D-Arg ${ }^{2}$ ) from Bachem (Germany). Phosphoric acid and ammonia solution were of pure grade. All chemicals were used as received. Distilled water was used for all preparations. All NPs had a PDI $<0.5$ [PDI $=$ polydispersity index, describing the variation in size].

\section{Histological staining (HE staining)}

Liver slices were left for 10 min in Mayer's Hämalaun and washed several times with distilled water and $\mathrm{HCl}-\mathrm{ethanol}$ ( $25 \%$ hydrochloric acid and $70 \% \mathrm{EtOH}, 1: 50)$. Then, the slices were left for $3 \mathrm{~min}$ in Eosin, 2 min in distilled water, in 70, 80 and $96 \%$ EtOH each 2 min and two times in $100 \% \mathrm{EtOH}$ for $5 \mathrm{~min}$. For the last step, the slices were dipped in Histoclear (Roth, Karlsruhe, Germany) three times for $5 \mathrm{~min}$.

\section{Hot plate test}

Analgesia was studied as a functional indicator for showing that NPs had successfully crossed the BBB. To this end, the rats were placed on a hot plate (model DS 37 socrel, 57 ${ }^{\circ} \mathrm{C}$ ), and the time until onset of licking or jumping (hot plate latency, maximum $30 \mathrm{~s}$ ) was recorded as a measure of analgesia. The hot plate latency was determined 5, 15, 30, 45, 90, 120, 180 and 240 min after iv injection of Kyotorphin-loaded NP.

\section{Statistic}

Statistical significance was determined in SPSS 21 by a one-way ANOVA and subsequent posthoc Tukey comparison.

\section{Results}

\section{Toxicity studies}

In vitro-We found differences between cell lines regarding the sensitivity towards NP incubation. HEK cells showed a decreased viability even at lower concentrations of NPs in the medium than HeLa cells (Fig. 2), starting already from $60 \mu \mathrm{g} / \mathrm{ml}$ of Lutrol-SDS NPs. In HEK cells, all NP variations led to viability values significantly below the LD50 threshold (middle lethal dose which caused $50 \%$ cell death) at higher concentrations. Especially, Lutrol-SDS-PBCA NPs, led to a dose-dependent damage in HeLa cultures $(p<0.01$ highly significant). Although absolute values differed between HeLa and HEK cells, the relative susceptibility towards the NP variations was consistent: NPs fabricated with Lutrol-SDS were most detrimental in both systems, and NPs produced with dextran showed the lowest 
toxicity. First significant differences $(p<0.01)$ between the effects of NP variations on viability were observed at concentrations of $60 \mu \mathrm{g} / \mathrm{ml}$ in HeLa and of $40 \mu \mathrm{g} / \mathrm{ml}$ in HEK cells for Lutrol-SDS NPs. Using Tween 80 as a tenside seems to be rather stressful for cells, but in combination with dextran, the cultures tolerated such NPs very well. Also NPs with LutrolDextran and DEAEDextran exhibited a high safety margin.

In vivo-To analyse the neurotoxic effect of chronic PBCA NP exposure, we injected two different NP solutions once per week for 4 weeks; quantified survival, cell size and fluorescence intensity of the RGC; and compared the results with a negative control (trauma-induced neuronal damage) and a sham control group (injection of fluorescent dye only; no BBB passage). Throughout the whole experiment, we monitored the behaviour and the appearance of the rats during daily animal care.

There was no difference in the weights of the animals amongst these four groups (Fig. 3). During normal handling, the rats did not show any abnormal behaviour like aggression, piloerection, porphyrin discharge, hunchback posture or thigmotaxis. At the end of the experiment, the heart, liver, kidney and brain were harvested, and after histological processing, microscopic examination of the tissue was performed. In none of the organs obvious morphological abnormalities were observed. The photomicrographs of liver tissue in Fig 4 serve as an example: here, no differences in histological appearance were noted after staining between tissues from rats injected with NPs (as proven by the intensive fluorescence in the tissue) and those without NP exposure.

When quantifying neuronal survival in the retina of living animals with ICON, we detected no difference in the number of RGC after NP application compared to the sham control group which received only injections of the fluorescent dye (no NPs). As expected, in the ONC group, $80 \%$ of RGC had died by the end of the experiment (Figs. 5, 6), and the neuronal survival was significantly lower than in the NP-treated rats and the sham control (fluorescent dye only).

In addition, we studied the changes in soma size of the RGC bodies and cell fluorescence. Because of technical reasons, we were unable to collect data from the fourth ICON measurement for these two parameters, and due to the optic nerve lesion, there were not enough surviving neurons present to allow for quantification of average size and fluorescence intensity in the ONC animals at the ICON 3 data collection time point.

Figure 7 demonstrates the decrease of soma size in all groups compared to baseline. There was no significant difference between groups at the first ICON analysis. At ICON 2, rats injected with Tween 80 NP showed significantly ( $p<0.1$ marginal significance) less shrinkage than rats from the other groups. ICON 3 revealed that Tween80-SDS animals were back to baseline values and different from the neuronal size of the sham control group $(p<0.1)$. While, in the sham control (Rho123) and Tween-80 NP groups, the cell size was relatively stable over time, the neurons in the ONC group were shrinking, and Tween80SDS NP-treated cells recovered normal size after a significant decrease. 
The significant differences in neuronal fluorescence intensity between groups and over time are shown in Fig. 8. Control animals injected with fluorescent dye only (sham control) show some significant decreases in fluorescence intensity over time. At ICON 3, we found no significant differences between NP-treated animals and the sham control. However, the temporal pattern is different: especially at ICON 2, the neurons of the NP-treated animals were more fluorescent than in sham controls. There was a steady decrease in fluorescence intensity of the ONC negative control group, which was most pronounced at ICON 2.

\section{Analgesia study}

Hot plate test-As in previous studies (Schroeder and Sabel 1996; Kreuter et al. 1995; Alyautdin et al. 1995), we found that administration of drug loaded PBCA NPs increased pain threshold. This shows that the analgesic agent reached the brain when delivered by NPs. Kyotorphin alone, i.e. non-bound to NPs at various doses, had no analgesic effects after iv injection. The iv application of Tween80 PBCA NPs incorporated with Kyotorphin significantly enhanced hot plate latency $(p<0.05)$ at $15 \mathrm{~min}$ after iv application compared with Kyotorphin alone (Fig. 9). When Kyotorphin was adsorbed onto Tween-PBCA NPs, no change of analgesia could be demonstrated compared with the Kyotorphin-alone-treated rats. Interestingly, a significant enhancement $(p<0.05)$ of analgesia was found at $15 \mathrm{~min}$ after application when NPs were incorporated with Kyotorphin compared to the adsorbed Kyotorphin, i.e. incorporation is the more effective method.

\section{Discussion}

There is an increasing interest for using NPs as drug delivery systems over the last decade. Because the BBB is a hurdle for CNS drug development, NPs may be one method to overcome this barrier. However, the future use of NPs as drug delivery systems into the CNS requires a better understanding about their potential neurotoxicity. To investigate this issue, we have now tested NPs in cell culture systems and - as an innovative approachused in vivo confocal neuroimaging (ICON). ICON is a unique method to analyse survival and morphology of retrogradely labelled retinal ganglion cells in living mammals (Sabel et al. 1997, Prilloff et al. 2010; Henrich-Noack et al. 2012). By injecting iv NPs and observing the effects of the particles on the fluorescent retinal ganglion cells, we were able to study the neurotoxicity of NPs after their passage through the blood-retina barrier (BRB) in real time for several weeks. The BRB is a valid model of the BBB which is supported by theoretical considerations and experimental data: (i) ontogenetically, the retina is CNS tissue; it is a protrusion of brain tissue during development, and retinal cells are mostly CNS neurons; (ii) the function of the BRB and BBB are very similar, if not identical, as the passages of different pharmacological active compounds are quite similar in the BBB and BRB (Steuer et al. 2004). (iii) It was also shown that in the retina—as in other parts of the brain—-tightjunctions are present, and BRB and BBB both have the typical efflux-systems: permeabilityglycoprotein and multidrug-resistant protein (Steuer et al. 2005). Thus, BRB is a valid BBB model with the advantage that it permits the use of the ICON method to visualise neurons in the CNS (for simplicity, we refer to the BRB as 'BBB' from here on). 
Cell culture models are regularly used to study nanoparticle toxicity (Hussain et al. 2005, Kante et al. 1982, Couvreur et al. 1982) with the assumption that they simulate adequately the in vivo situation. In a first step, we therefore tested the cytotoxic effects of PBCA NP in two cell culture systems (HeLa and HEK) using the MTT viability test. Although the MTTassay is the most commonly used for testing nanotoxicity, false positive results have been described caused by molar extinction of the NPs (Dhawan and Sharma 2010; Sharma et al. 2012; Love et al. 2012). However, this problem applies more to silicone NPs, graphene and carbon nanotubes, but it may not be so crucial when comparing polymeric NPs with different tensides as we did. The in vitro test permits the screening of many different NP dosages to estimate possible toxicity. HeLa cells are human cervical carcinoma cells and more robust than HEK cells which explains the difference between cell lines. Some authors recommend more sophisticated in vitro test systems (e.g. co-cultres; Love et al. 2012), but in our experiments, the cell culture was only used as a screening pre-test before in vivo evaluation. Therefore, there was no reason to carry out more sophisticated in vitro tests.

None of our NP variations decreased cell viability compared to controls across a wide range of concentrations. However, both cell lines consistently revealed that NPs fabricated with Lutrol-SDS were-at higher concentrations-more toxic than other NP variations. As Lutrol-Dextran or Tween80-Dextran NPs, on the other hand, induced significantly less damage, we conclude that especially SDS should be avoided when producing NPs for human applications. As Tween 80 has repeatedly been shown to facilitate BBB passage of PBCA NPs, it is widely used as a surfactant. Our cell culture model indicates that Tween80 NPs, however can decrease cell viability already at lower concentrations than Tween80Dextran NPs.

Having specified the NP/tenside combinations which may be toxic when applied to biological systems, we studied different particle systems in vivo. It has been repeatedly criticised that in vitro studies alone are insufficient and that especially neurotoxicity has hardly been tested in vivo so far. This issue is crucial in the field of nanotoxicity as in nature there are no 'bare nanoparticles' and the biomolecule corona (e.g. after contact with blood) on the nanoparticle surface will be decisive for the biological (and toxicological) outcomes of nanoparticle interaction with biological systems (Elsaesser and Howard 2012; Zhao and Castranova 2011; Sharma et al. 2012; Nyström and Fadeel 2012). Our own work (HenrichNoack et al. 2012) and that by others (Kreuter et al. 1997, 2002, Weiss et al. 2007; Schroeder et al. 1998) demonstrated that PBCA NPs manufactured with the surfactant Tween 80 can cross the BBB. We therefore fabricated NPs with Tween80, dispersed them in physiological solution and injected the NPs once per week for 4 weeks in the tail veins of the rats. In contrast to Lam et al. 2004 who reported tissue damage in mice resulting in granuloms, we did not find any general disturbances (such as weight loss) and no adverse tissue reactions in different organs were observed when assessing the morphology with standard histological analysis. Moreover, we could not detect any neuronal death over a period of 4 weeks when quantifying neuronal survival with in vivo imaging. Using ICON, we repeatedly followed the fate of single neurons in vivo over time. The ICON test, which is very sensitive to reveal neuronal cell death even of single cells, strongly indicates that PBCA NPs—even with Tween 80 coating — are safe and can be used as drug carriers in mammals. We did not observe a single cell dying in vivo in response to NP exposure. 
However, there are some interesting details when looking at the ICON results regarding the morphology of the neurons. In control animals (receiving only the fluorescent dye, no BBB passage), the average cell size of the retrogradely labelled retinal ganglion cells appeared to become smaller after baseline measurement, but the soma diameter remained stable over time. As expected, RGCs in animals subjected to ONC are larger soon after the lesion (ICON 1; swelling) but then shrink (ICON 2) before they disappear (ICON 3) (HenrichNoack et al. 2013). The neurons of rats which received NP injections are, interestingly, on average larger than neurons in control animals without NP injections. This non-lethal swelling may be due to (i) slight osmotic dysregulation due to stress (cellular oedema) or (ii) due to increased activity/swelling of lysosomes. Ku et al. 2010 have shown that polymeric NPs can be located within neurons, and NPs may colocalise with lysosomes and induce significant dilatation (Fröhlich 2012) which may be the cause of increased soma size.

We also quantified the fluorescence intensity of the retinal neurons, and our findings are also in line with the assumption that the PBCA NPs are non-toxic. In lesioned animals, the fluorescence intensity decreases over time, probably due to degeneration-induced membrane leakage of the damaged retinal ganglion cells. Fluorescence intensity of the control animals' (no NP injection) neurons also decreases over time albeit less than that in damaged neurons. NP-injected animals show the same fluorescence intensity of retinal ganglion cells as the control animals during the last measurement time point (ICON 3). This indicates that there are no membrane disruption and no leakage of dye from neurons after NP exposure. This is an important finding as there have been concerns that NPs, especially polymeric NPs, may damage membranes and disrupt barriers (Elsaesser and Howard 2012; Sharma et al. 2012; Nunes et al. 2012; Nyström and Fadeel 2012). In this respect, our results are rather reassuring as they indicate that there are no holes in the cells, and PBCA NPs can be safely applied in biological systems. The reason for the higher values of neuronal fluorescence intensities measured at ICON 2 for animals injected with NPs is unclear. We hypothesise that either there are some differences in intracellular $\mathrm{pH}$ (fluorescence intensity of fluorescein dextran is ph-dependent) or the accumulation of NPs in lysosomes competes with the cellular processing of the fluorescent dye.

In an additional experiment, we tested whether NPs-in the concentration range tested for toxic effects - can carry sufficient amount of a drug into the brain to induce pharmacological effects. To this end, we fabricated PBCA NPs to which Kyotorphin was incorporated or adsorbed, injected them into the rat's tail vein and monitored the analgesic latency with the hot plate paradigm. We found that administration of Tween80 PBCA NPs with incorporated Kyotorphin showed a significant analgesic activity in this behavioural test. This result confirms that PBCA NPs in nontoxic concentrations can be used as drug carriers for CNS applications. However, our findings are at first glance partially in contrast to prior observation by others (Alyautdin et al. 1995; Kreuter et al. 1995; Schroeder and Sabel 1996) who showed analgesic effects of PBCA NPs with adsorbed drugs. However, in our experiments, the formulation with adsorbed Kyotorphin had no effect whatsoever. This difference may be explained by the variations in the production protocols. Moreover, in our former work, we also detected that PBCA NPs deliver adsorbed fluorescent dyes with much lower efficiency into the brain than those with incorporated fluorescent dyes (not shown). 
In summary, we demonstrated that in vivo PBCA NPs do not induce neuronal death in pharmacologically effective concentrations, even when coated with surfactants that are adverse to viability in cell cultures. From these in vivo observations, we conclude that PBCA NPs are a non-toxic tool for drug delivery into the CNS.

\section{Acknowledgments}

The authors thank Uta Werner for excellent technical assistance. Nadine Voigt was supported by a grant from Ottovon-Guericke University. Petra Henrich-Noack received funding from the Sybille Assmus-Foundation.

\section{References}

Ai J, Biazar E, Jafarpour M, Montazeri M, Majdi A, Aminifard S, Zafari M, Akbari HR, Rad HG. Nanotoxicology and nanoparticle safety in biomedical designs. Int J Nanomed. 2011; 6:1117-1127.

Alyautdin RN, Gothier D, Petrov VE, Kharkevich DA, Kreuter J. Analgesic activity of the hexapeptide dalargin adsorbed on the surface of polysorbate 80-coated poly(butylcyanoacrylate) nanoparticles. Eur J Pharm Biopharm. 1995; 41:44-48.

Couvreur P, Kante B, Grislain L, Roland M, Speiser P. Toxicity of polyalkylcyanoacrylate nanoparticles. I. Doxorubicin-loaded nanoparticles. J Pharm Sci. 1982; 71:790-792. [PubMed: 7120064]

De Jong WH, Borm PJ. Drug delivery and nanoparticles: applications and hazards. Int J Nanomedicine. 2008; 3:133-149. [PubMed: 18686775]

Dhawan A, Sharma V. Toxicity assessment of nanomaterials: methods and challenges. Anal Bioanal Chem. 2010; 398:589-605. [PubMed: 20652549]

Elsaesser A, Howard CV. Toxicology of nanoparticles. Adv Drug Deliv Rev. 2012; 64:129-137. [PubMed: 21925220]

Fröhlich E. The role of surface charge in cellular uptake and cytotoxicity of medical nanoparticles. Int J Nanomedicine. 2012; 7:5577-5591. [PubMed: 23144561]

Gabathuler R. Approaches to transport therapeutic drugs across the blood-brain barrier to treat brain diseases. Neurobiol Disease. 2010; 37:48-57.

Henrich-Noack P, Prilloff S, Voigt N, Jin J, Hintz W, Tomas J, Sabel BA. In vivo visualisation of nanoparticle entry into the central nervous system tissue. Arch Toxicol. 2012; 86:1099-1105. [PubMed: 22422342]

Henrich-Noack P, Voigt N, Prilloff P, Fedorov A, Sabel BA. Transcorneal electrical stimulation alters morphology and survival of retinal ganglion cell after axonal damage. Neurosci Lett. 2013; 543:16. [PubMed: 23523651]

Hussain SM, Hess KL, Gearhart JM, Geiss KT, Schlager JJ. In vitro toxicity of nanoparticles in BRL 3A rat liver cells. Toxicol In Vitro. 2005; 19:975-983. [PubMed: 16125895]

Kante B, Couvreur P, Dubois-Krack G, De Meester C, Guiot P, Roland M, Mercier M, Speiser P. Toxicity of polyalkylcyanoacrylate nanoparticles. J Pharm Sci. 1982; 71:786-790. [PubMed: 7120063]

Kreuter J, Alyautdin RN, Kharkevich DA, Ivanov AA. Passage of peptides through the blood-brain barrier with colloidal polymer particles (nanoparticles). Brain Res. 1995; 674:171-174. [PubMed: 7773690]

Kreuter J, Petrov VE, Kharkevich DA, Alyautdin RN. Influence of the type of surfactant on the analgesic effects induced by the peptide dalargin after its delivery across the blood-brain barrier using surfactant-coated nanoparticles. J Control Release. 1997; 49:81-87.

Kreuter J, Shamenkov D, Petrov V, Ramge P, Cychutek K, Koch-Brandt C, Alyautdin R. Apolipoprotein mediated transport of nanoparticle-bound drugs across the blood-brain barrier. $\mathrm{J}$ Drug Target. 2002; 10:317-325. [PubMed: 12164380]

Ku S, Yan F, Wang Y, Sun Y, Yang N, Ye L. The blood-brain barrier penetration and distribution of PEGylated fluorescein-doped magnetic silica nanoparticles in rat brain. Biochem Biophys Res Commun. 2010; 394:871-876. [PubMed: 20206605] 
Lam CW, James JT, McCluskey R, Hunter RL. Pulmonary toxicity of single-wall carbon nanotubes inmice 7 and 90 days after intratracheal instillation. Toxicol Sci. 2004; 77:126-134. [PubMed: $14514958]$

Love SA, Maurer-Jones MA, Thompson JW, Lin YS, Haynes CL. Assessing nanoparticle toxicity. Annu Rev Anal Chem. 2012; 5:181-205.

Nunes A, Al-Jamal KT, Kostarelos K. Therapeutics, imaging and toxicity of nanomaterials in the central nervous system. J. Control Release. 2012; 161:290-306. [PubMed: 22512901]

Nyström AN, Fadeel B. Safety assessment of nanomaterials: implications for nanomedicine. J. Control Release. 2012; 161:403-408. [PubMed: 22306428]

Oberdörster G, Sharp Z, Atudorei V, Elder A, Gelein R, Kreyling W, Cox C. Translocation of inhaled ultrafine particles to the brain. Inhal Toxicol. 2004; 16:437-445. [PubMed: 15204759]

Prilloff S, Fan J, Henrich-Noack P, Sabel BA. In vivo confocal neuroimaging (ICON): non-invasive, functional imaging of the mammalian CNS with cellular resolution. Eur J Neurosci. 2010; 31:521528. [PubMed: 20105236]

Sabel BA, Engelmann R, Humphrey MM. In vivo confocal neuroimaging of CNS neurons (ICON). Nat Med. 1997; 3:244-247. [PubMed: 9018248]

Sautter J, Sabel BA. Recovery of Brightness discrimination in adult rats despite progressive loss of retrogradely labelled retinal ganglion cells after controlled optic nerve crush. Eur J Neurosci. 1993; 5:680-690. [PubMed: 8261140]

Sautter J, Schwartz M, Duvdevani R, Sabel BA. GM1 ganglioside treatment reduces visual deficits after graded crush of the rat optic nerve. Brain Res. 1991; 565:23-33. [PubMed: 1773355]

Schroeder U, Sabel BA. Nanoparticles, a drug carrier system to pass the blood-brain barrier, permit central analgetic effects of i.v. dalargin injections. Brain Res. 1996; 710:121-124. [PubMed: 8963650]

Schroeder U, Sommerfeld P, Ulrich S, Sabel BA. Nanoparticle technology for delivery of drugs across the blood-brain-barrier. Eur J Pharm Sci. 1998; 87:1305-1307.

Scientific Committee on Emerging and Newly Identified Health Risks (SCENIHR): Modified Opinion (after public consultation). on the appropriateness of existing methodologies to assess the potential risk associated with engineered as adventitious products of nanotechnologies. European Commission Health and Consumer Protection Directorate-General, Directorate C-Public Health and Risk Management, C7-Risk assessment; 10th Plenary Meeting; 10th of March 2006; 2006.

Sharma A, Madhunapantula SV, Robertson GP. Toxicological considerations when creating nanoparticle based drugs and drug delivery systems? Expert Opin Drug Metab Toxicol. 2012; 8:47-69. [PubMed: 22097965]

Steuer H, Jaworski A, Stoll D, Schlosshauer B. In vitro model of the outer blood-retina barrier. Brain Res Protoc. 2004; 13:26-36.

Steuer H, Jaworski A, Elger B, Kaussmann M, Keldenich J, Schneider H, Stoll D, Schlosshauer B. Functional characterization and comparison of the outer blood-retina barrier and the blood-brain barrier. Invest Ophthalmol Vis Sci. 2005; 46:1047-1053. [PubMed: 15728564]

Weiss CK, Ziener U, Landfester K. A route to nonfunctionalized and functionalized poly- $(n-$ butylcyanoacrylate) nanoparticles: preparation in miniemulsion. Macromolecules. 2007; 40:928938.

Zhao J, Castranova V. Toxicology of nanomaterials used in nanomedicine. J Toxicol Environ Health Part B. 2011; 14:593-632. 


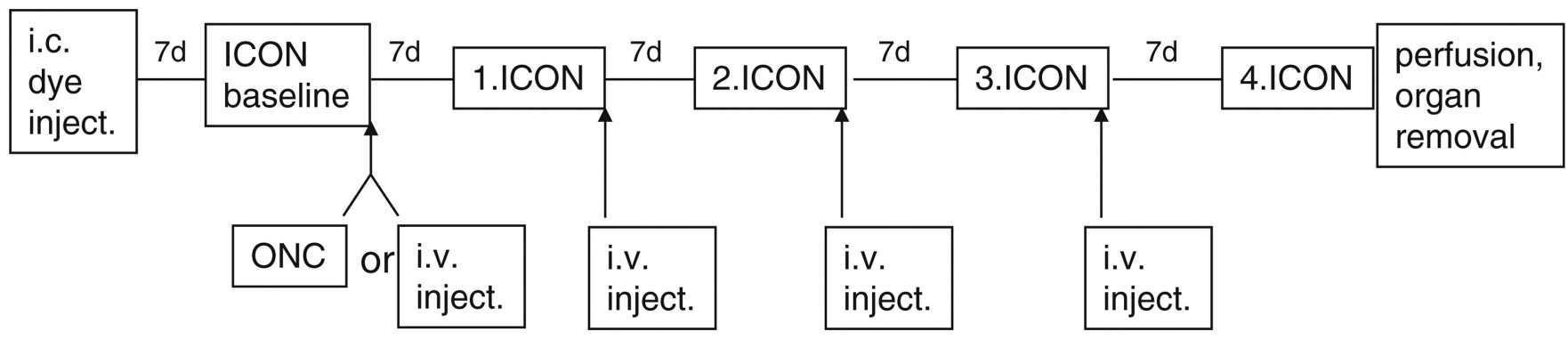

Fig. 1.

Experimental design of repeated NP exposures in vivo. Rats received an injection of the fluorescent tracer into the superior colliculus (ic). Baseline retinal imaging was performed 7 days later and directly afterwards animals were either subjected to optic nerve crush (ONC) or received an injection of a NP or Rho123 solution into the tail vein (iv). After 4 injections and 4 ICON sessions, animals were intracardially perfused, and liver, heart, kidney and brain harvested 
MTT HeLa
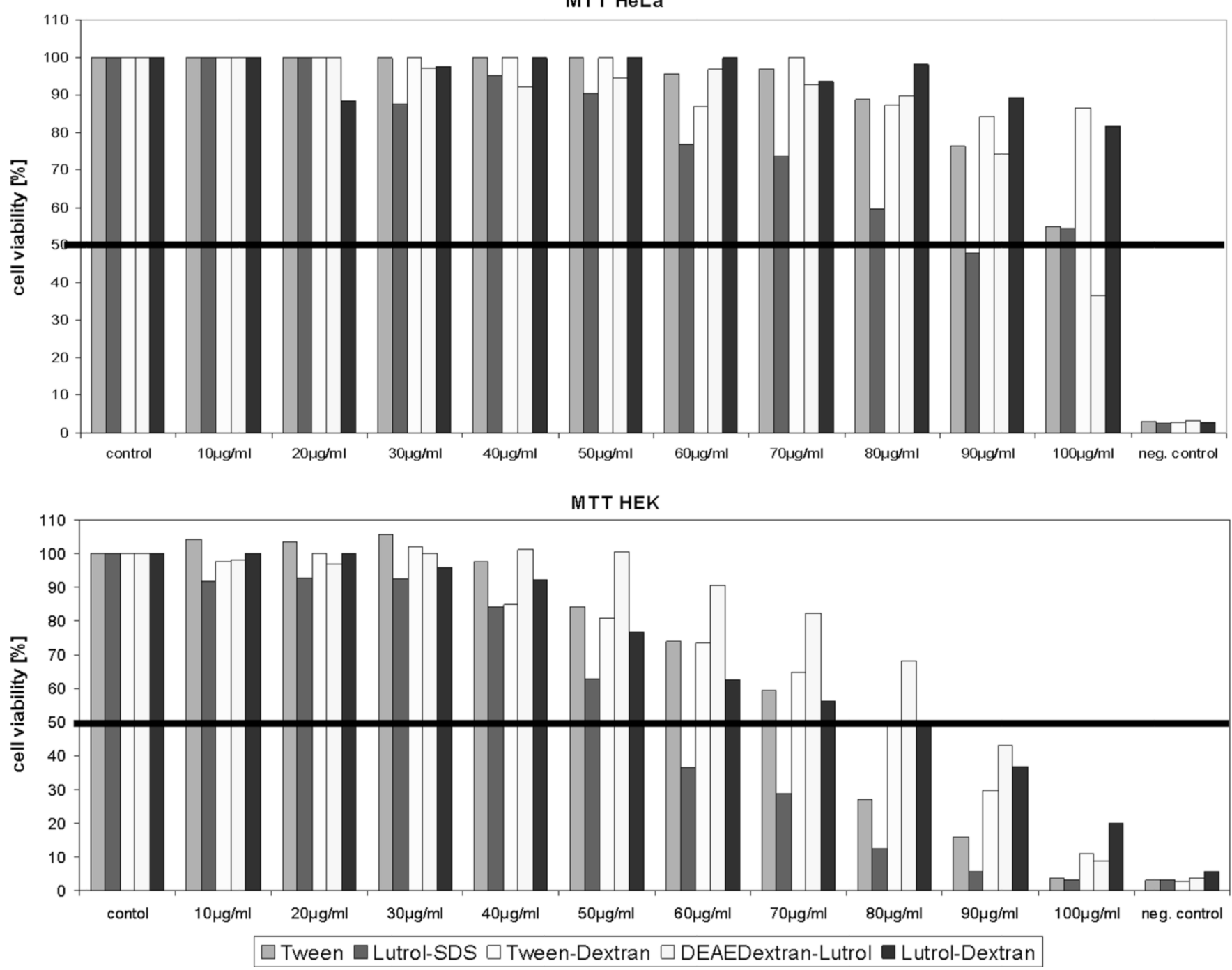

Fig. 2.

Cytotoxicity in MTT-test. The upper graph shows the results obtained with HeLa cells and lower graph data from HEK293 cells. The black line marks the middle lethal dose $\left(\mathrm{LD}_{50}\right)$, i.e. the NP dose which caused $50 \%$ death 


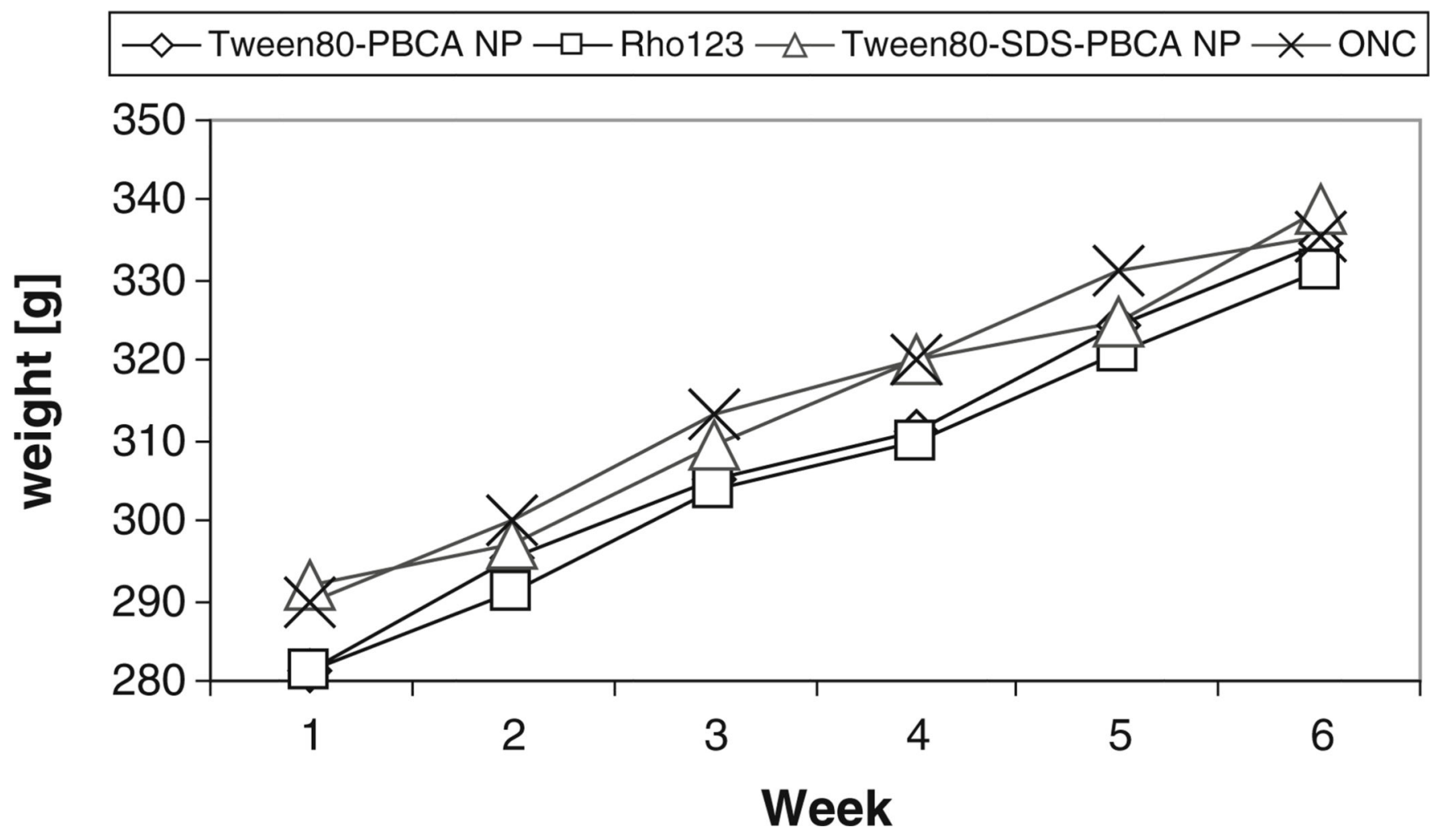

Fig. 3.

Weight monitoring during recurrent NP applications. This graph gives an overview of the animal's body weight during the period of the in vivo experiment. There is no significant difference between the four groups, indicating that the rats did not suffer from major health impairments 

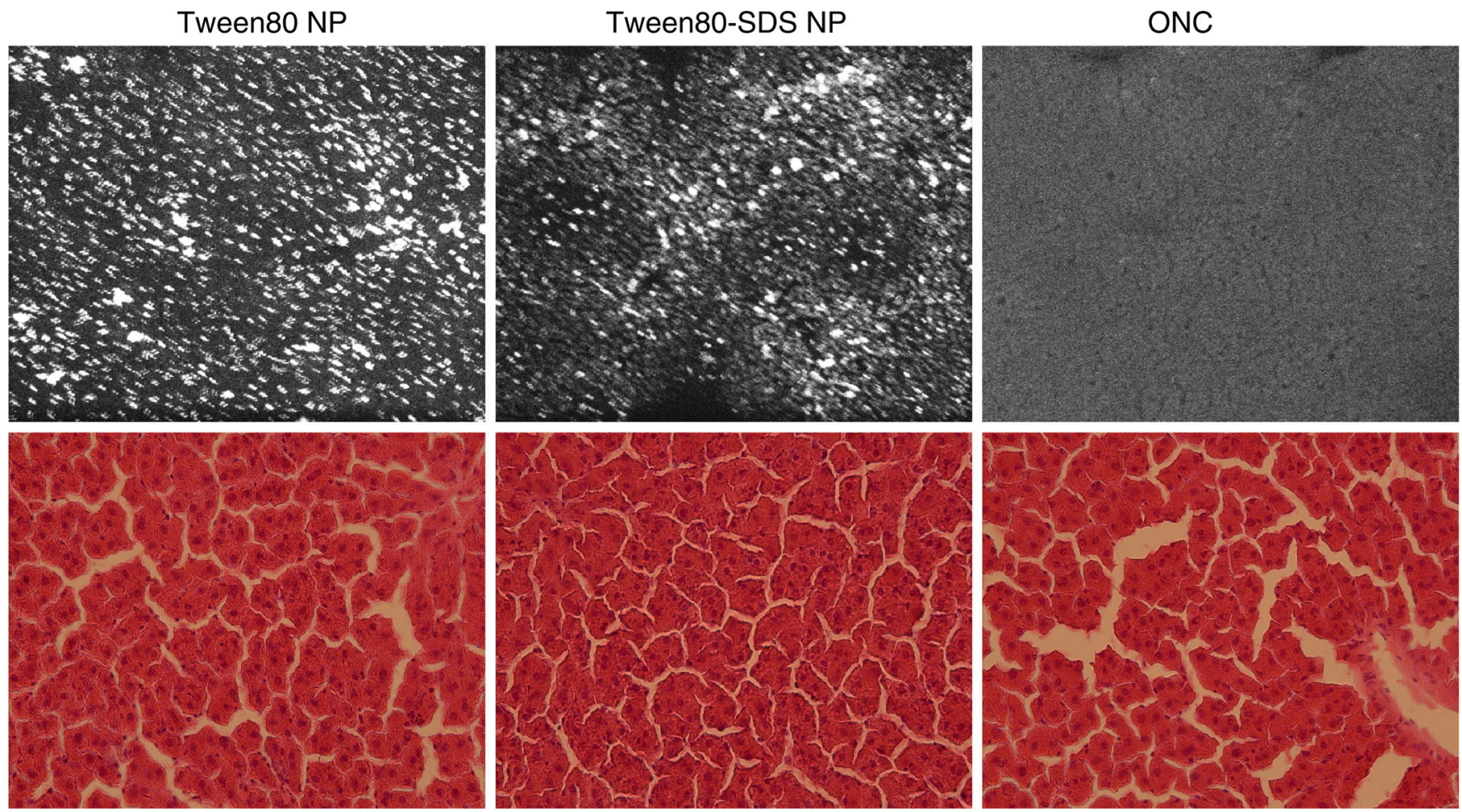

Fig. 4.

Morphological examination of liver. Although there is a massive fluorescent signal in the liver, indicating accumulation of PBCA NP in this organ, we do not see any abnormalities after histological processing (HE staining). For comparison, the very right panel is a photomicrograph of the tissue from an animal which did not receive any NP injections 


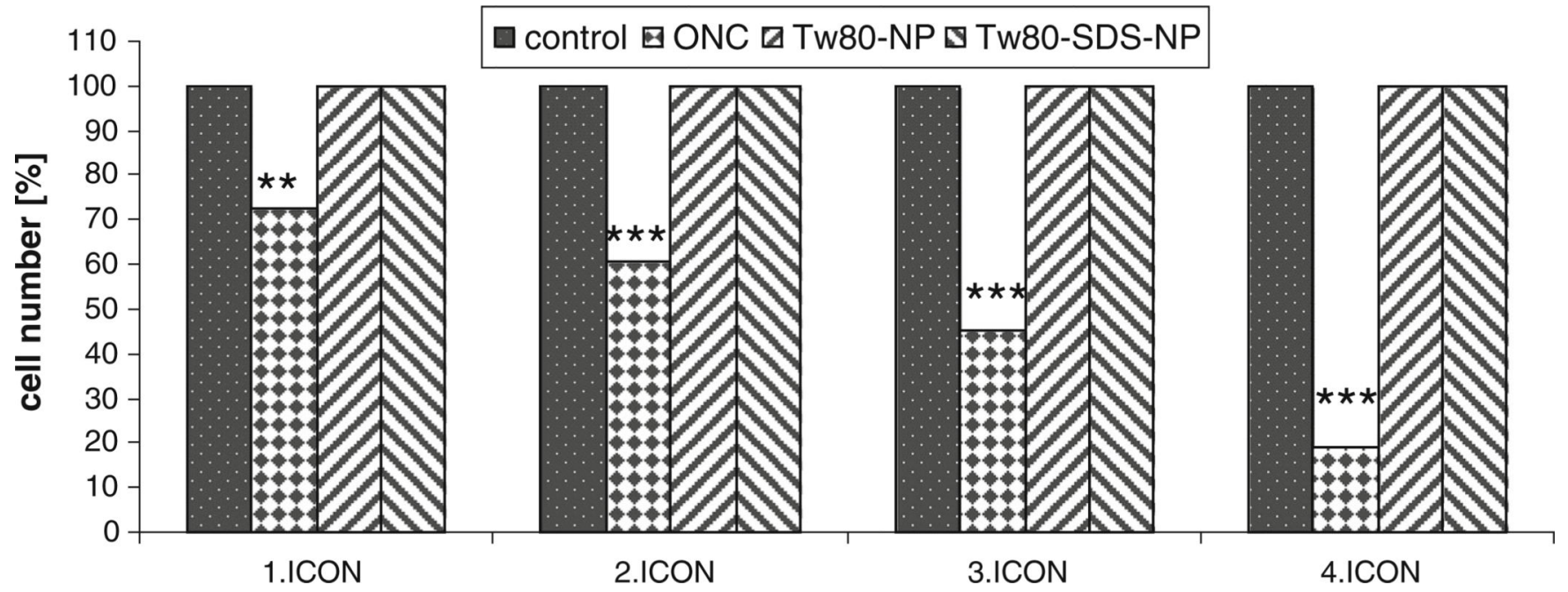

Fig. 5.

Constant number of retinal ganglion cells after NP application. This bar graph demonstrates that there is no decrease in the number of RGC after injection of NP. Only in the group with ONC-induced lesion, neuronal death—as expected—could be detected: $80 \%$ of the cells had died by the end of the experiment (one-way ANOVA in conjunction with posthoc Tukey test $* p<0.05, * * p<0.01$ and $* * * p<0.001$ ) 

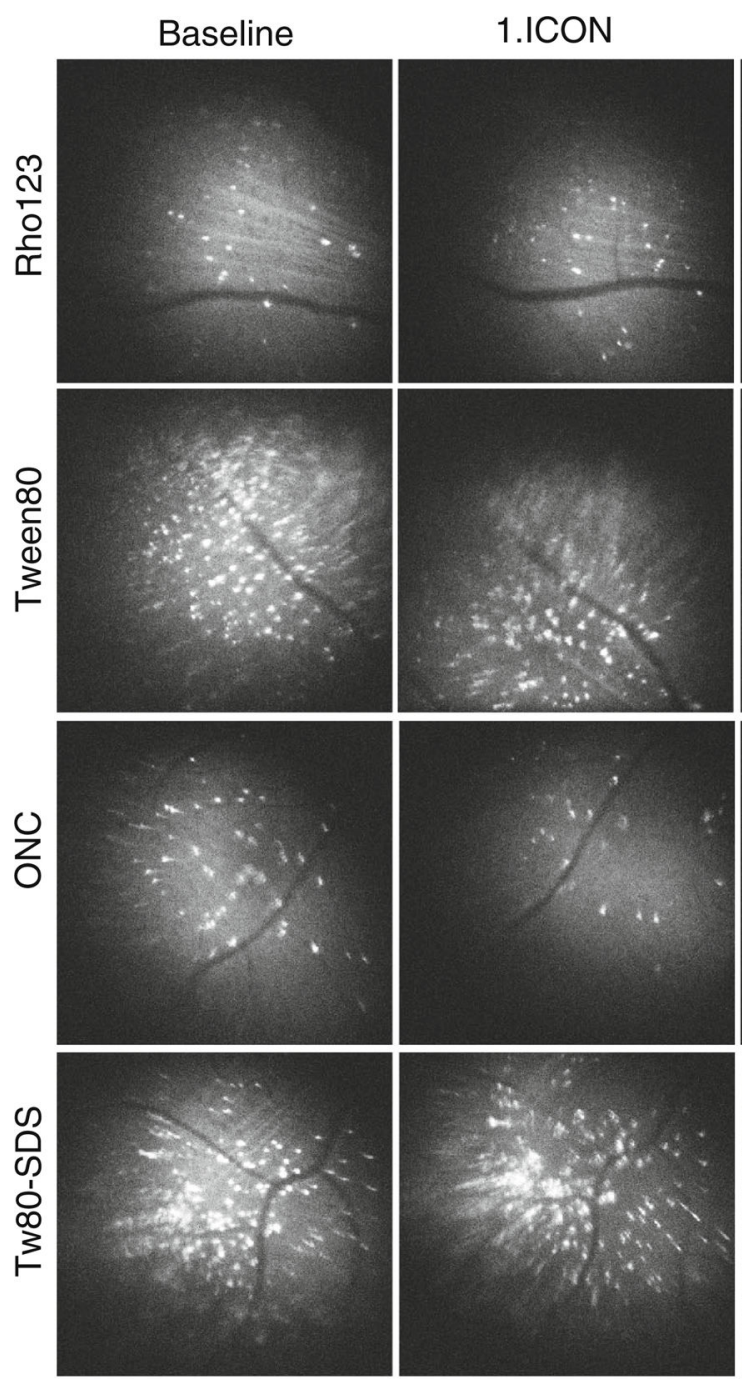

2.ICON
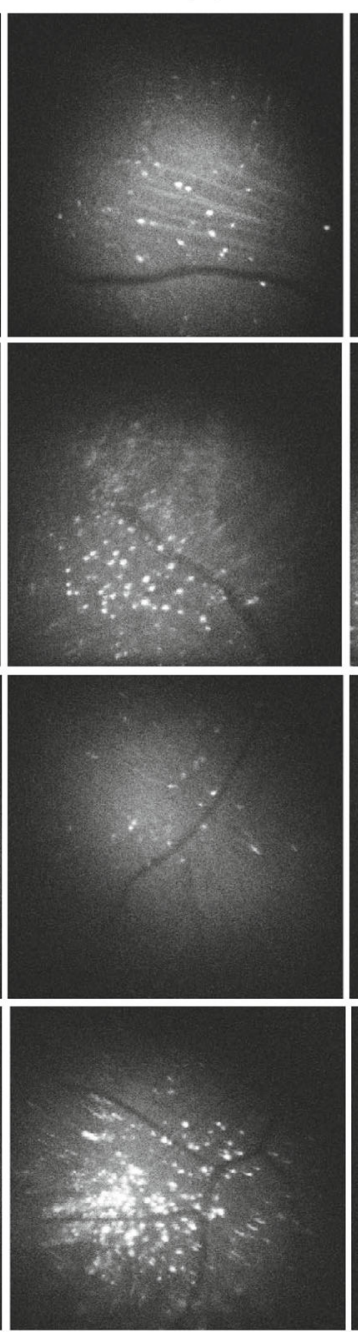

3.ICON
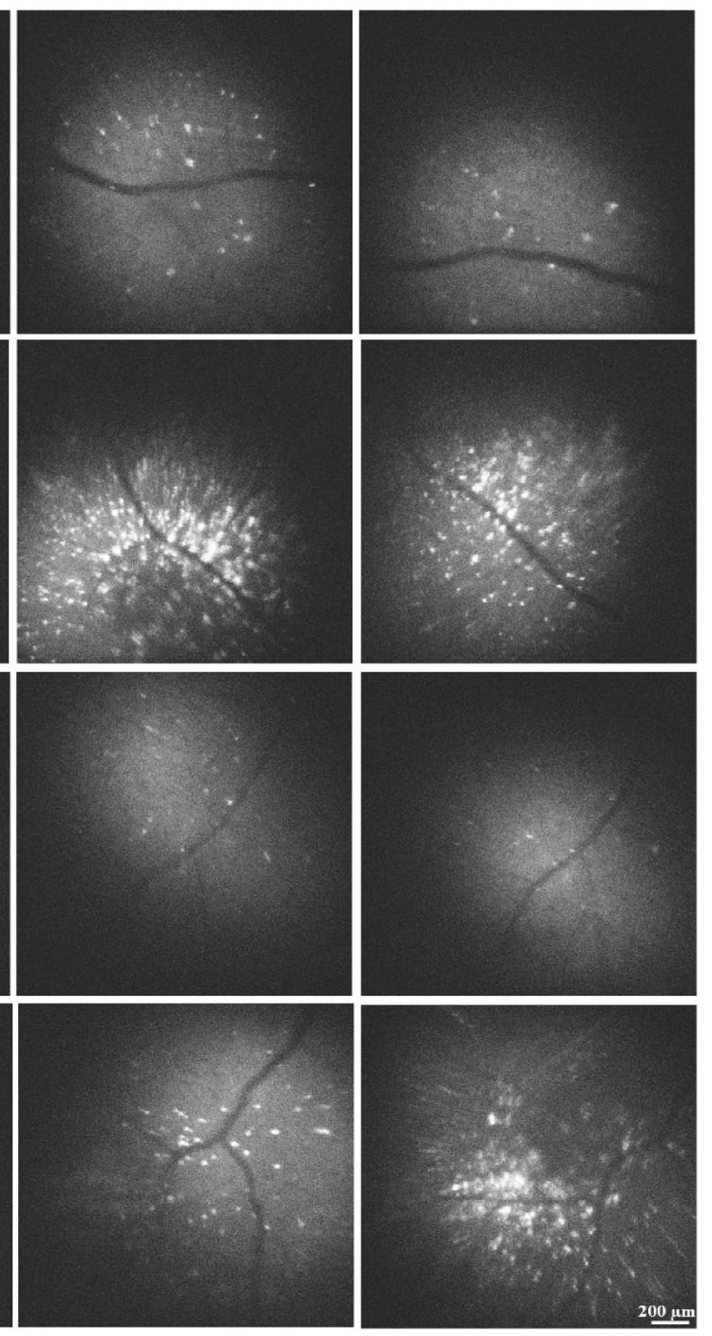

Fig. 6 .

Imaging overview. These photomicrographs illustrate fluorescently labelled retinal ganglion cells (RGCs) as imaged by the ICON technique. It shows that there is only a significant decrease in the number of RGC after ONC lesion, not after NP applications (Tween80 and Tw80-SDS). Bar $200 \mu \mathrm{m}$ 


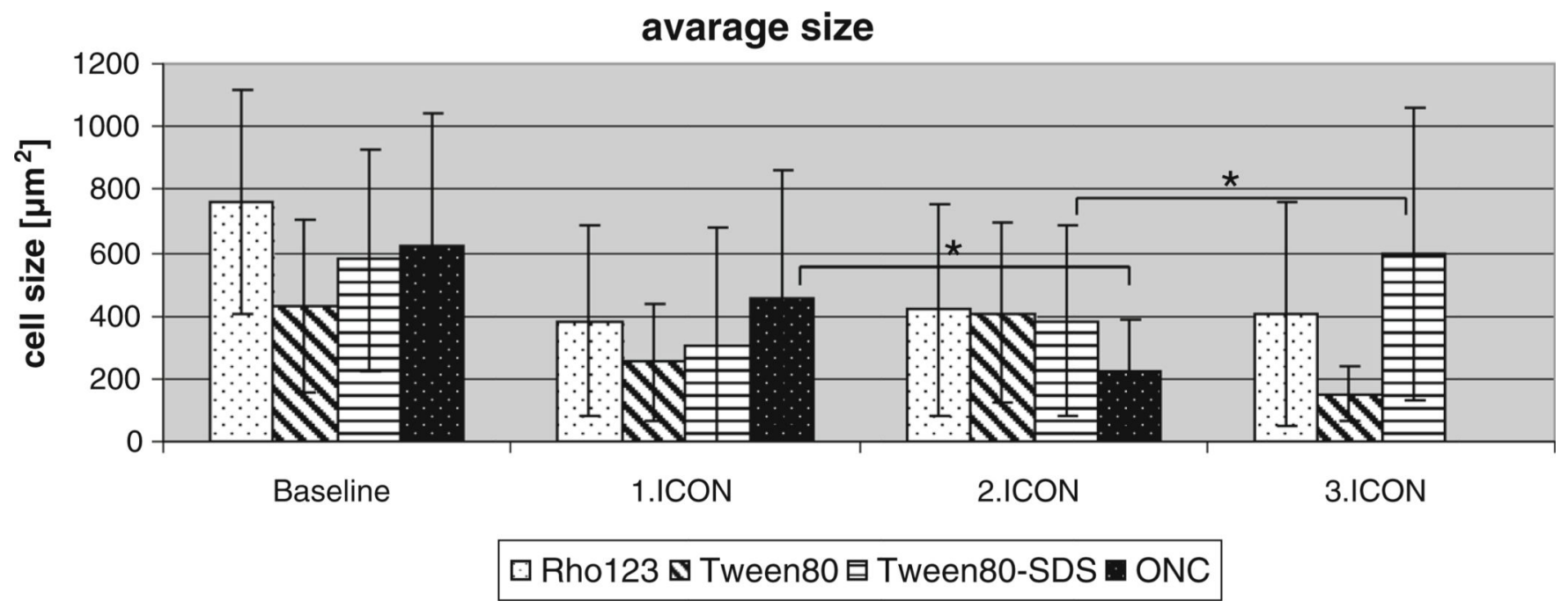

Fig. 7.

Quantification of retinal cell size in vivo with ICON. The bar graph shows the soma size of RGCs. In general, the animals injected with NPs exhibit less soma size difference than sham-control animals (only fluorescent dye injection and no NP injection) and lesioned animals (ONC optic nerve crush) (one-way ANOVA in conjunction with posthoc tukey test $* p<0.05)$ 


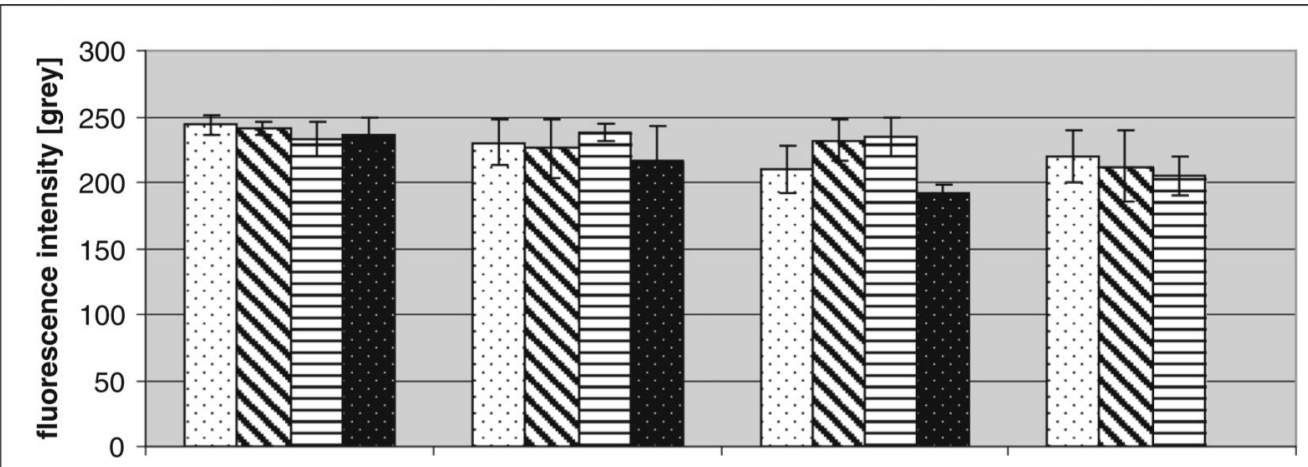

Baseline

1.ICON

2.ICON

3.ICON

口Rho123 వTween80 日Tween80-SDS aONC

\begin{tabular}{|l|c|l|l|l|l|l|}
\hline Statistic & Rho-Tw80 & Rho-ONC & Rho-Tw80SDS & Tw80-Tw80SDS & Tw80-ONC & ONC-Tw80SDS \\
\hline 1. ICON & & & & & & \\
\hline 2. ICON & $\star \star$ & & $\star$ & & $\star \star \star$ & $\star \star \star$ \\
\hline 3. ICON & & & & & & \\
\hline
\end{tabular}

\begin{tabular}{|l|c|c|c|}
\hline Statistic & 2.ICON-1.ICON & 3. ICON-1.ICON & 3. ICON-2.ICON \\
\hline Rho123 & $\star \star$ & & \\
\hline Tween80 & & & \\
\hline Tween80-SDS & & $\star \star \star$ & $*$ \\
\hline ONC & $\star \star$ & & \\
\hline
\end{tabular}

Fig. 8.

Change in fluorescence intensity in RGC. Fluorescence intensity differences compared to baseline show specific patterns after ONC and a transient influence of NP application (oneway ANOVA in conjunction with posthoc tukey test $* p<0.05, * * p<0.01, * * * p<0.001$ ) 


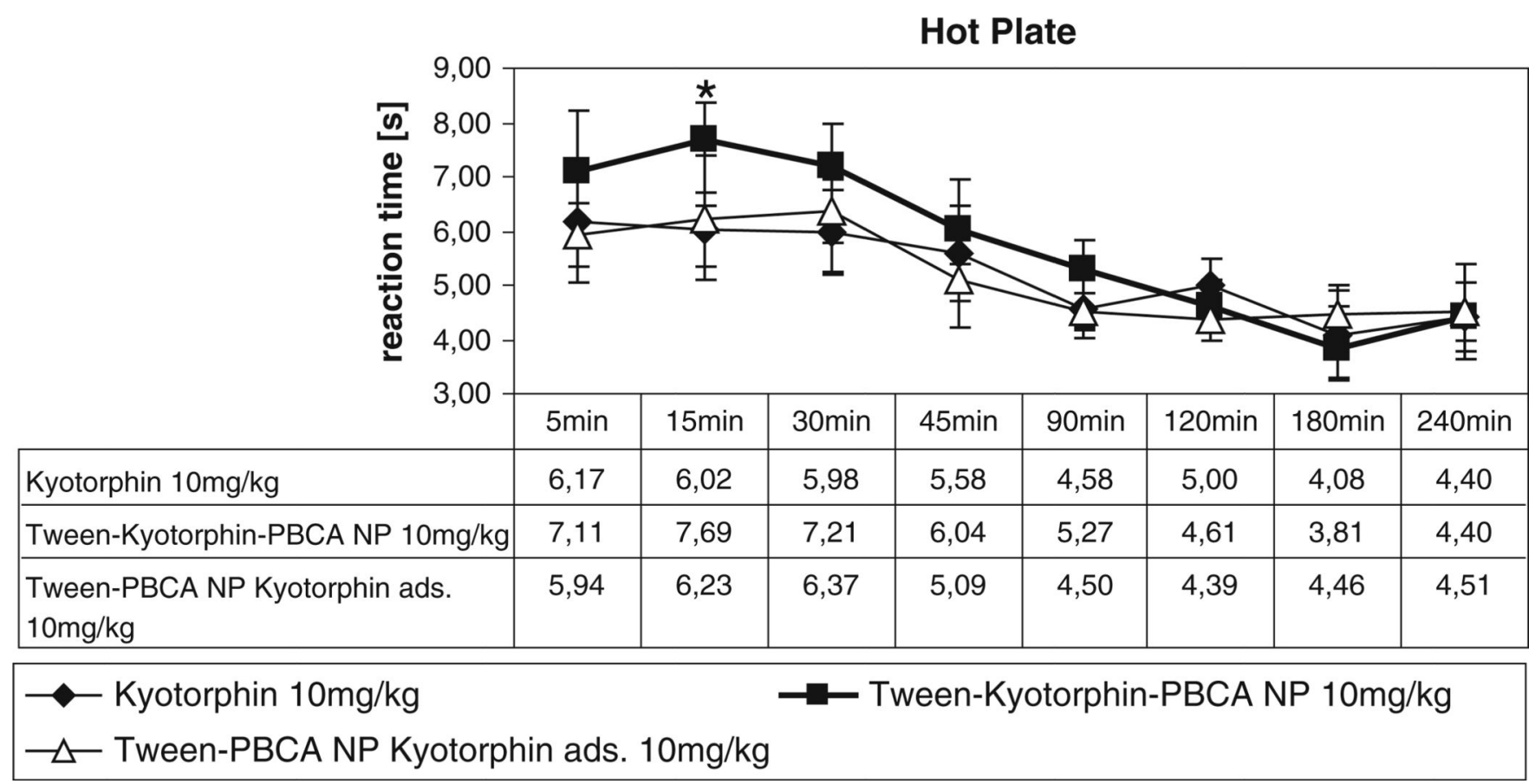

Fig. 9.

PBCA NP as potential candidates for drug delivery across the BBB. This figure demonstrates a significant increase of pain threshold after application of incorporated Kyotorphin-Tween-PBCA NP (one-way ANOVA in conjunction with posthoc tukey test $* p$ $<0.05)$ 


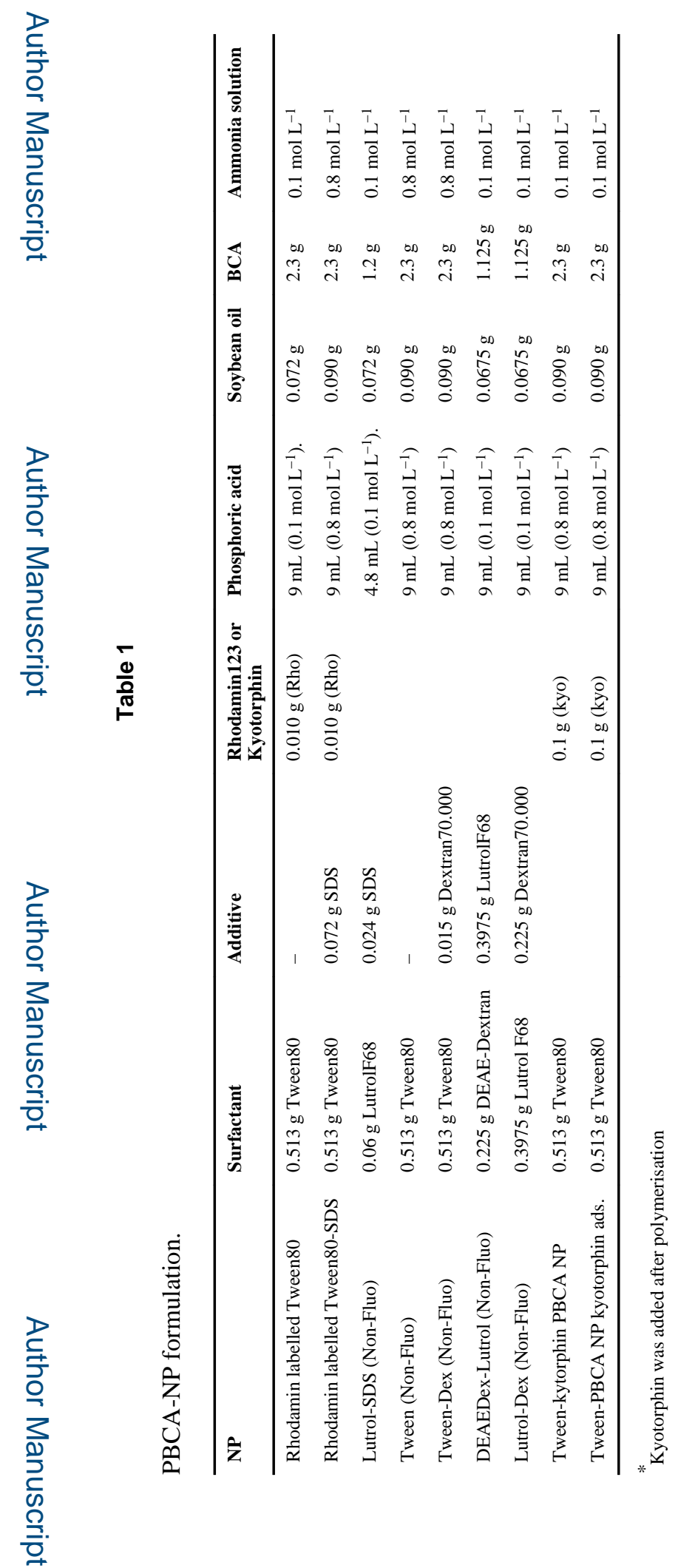



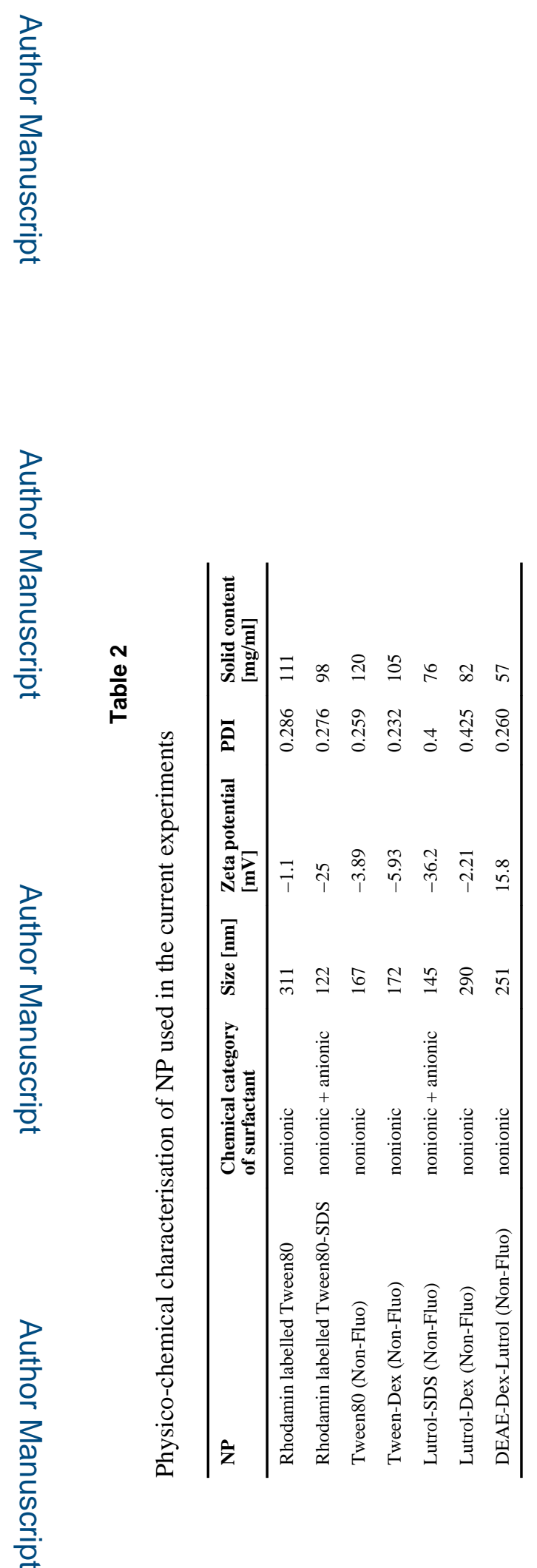

J Nanopart Res. Author manuscript; available in PMC 2015 September 27. 